\title{
Prevalence and Related Factors of Metabolic Syndrome in Beijing, China (Year 2017)
}

\author{
Aijuan Ma Kai Fang Jing Dong Zhong Dong \\ Beijing Center for Disease Prevention and Control, Beijing Research Center for Preventive \\ Medicine, Beijing, PR China
}

\section{Keywords}

Metabolic syndrome $\cdot$ Obesity $\cdot$ Risk factors $\cdot$ Waist circumference $\cdot$ Beijing

\begin{abstract}
Objective: Metabolic syndrome (MetS) is one of the major public health problems worldwide. The aim of this study was to investigate the prevalence and associated risk factors of MetS in Beijing to formulate targeted policies. Methods: Data from the 2017 Beijing Chronic Disease and Risk Factors Surveillance were used in this study, in which multistage stratified cluster sampling was adopted to collect a representative sample of 12,597 Beijing residents aged from 18 to 79 years. According to the definition of the International Diabetes Federation, the weighted prevalence of MetS and clustering of MetS components were estimated. The RaoScott adjusted $\chi^{2}$ test was used to test differences in MetS and components rates, and complex sampling unconditional logistic regression was used to explore influencing factors of MetS. Results: The prevalence of MetS was 25.59\% (95\% Cl 23.77-27.41), and the proportion of men and women was 30.53\% (95\% Cl 28.32-32.75) and 20.44\% (95\% Cl 18.29-22.58), respectively. The proportion of central obesity, high fasting plasma glucose, high triglyceride, low high-density lipoprotein cholesterol, and high blood pressure (BP) was 42.02, 27.96, 32.87, 27.25 , and $43.06 \%$, respectively. A total of $29.60 \%(95 \% \mathrm{Cl} 27.55-31.74)$ participants presented at least three components of MetS. The results from complex sampling unconditional logistic regression revealed that the risk factors for having MetS included being 45-59 years old, being 60-79 years old, being male, living in a rural area, education with junior middle school level, education with primary school or below level, harmful use of alcohol, inappropriate sleep time, and having an unhealthy waist-to-height ratio (WHtR); the OR values were 1.55 (95\% Cl 1.32-1.81), 1.94 (95\% Cl 1.62-2.31), 1.51 (95\% Cl 1.34-1.70), 1.27 (95\% Cl 1.06-1.52), 1.38 (95\% Cl 1.13-1.68), 1.44 (95\% Cl 1.13-1.84), 1.50 (95\% Cl 1.14-1.99), 1.23 (95\% Cl 1.10-1.37), and
\end{abstract}


Ma et al.: Metabolic Syndrome in Beijing

238.20 (95\% Cl 92.54-613.12), respectively. Conclusions: The prevalence of MetS is still in a rising trend in Beijing. Strategies aimed at prevention and control of high BP should be prioritized to reduce the occurrence of MetS. WHtR is more important to evaluate MetS. Health education and personalized lifestyle intervention should be promoted to keep a healthy $\mathrm{WHtR}$ and waist circumference. An appropriate sleep time should be kept, and harmful alcohol drinkers should limit or abstain from alcohol.

(C) 2020 The Author(s)

Published by S. Karger AG, Basel

\section{Introduction}

Metabolic syndrome (MetS) is characterized by a cluster of metabolic disorders, including abdominal obesity, hyperglycemia, dyslipidemia, and high blood pressure (BP) [1]. MetS has become a major public health problem worldwide [2]. It has been approved that MetS is associated with an increased risk of developing type 2 diabetes [3] and cardiovascular disease as well as with an increase in general mortality [4,5]. Major efforts are underway to detect, treat, and prevent MetS as a means of lowering the risk of diabetes and cardiovascular disease in the world [6]. It has been showed that the prevalence of MetS was $23.2 \%$ in 2005 for Beijing residents [7]. With the development of society and change in lifestyle, the prevalence of obesity, diabetes, and hypertension in 2014 for Beijing residents was $21.9,9.0$, and $34.9 \%$, respectively, which has increased by $3.8,1.1$, and $3.3 \%$, respectively compared to that in 2011 [8]. Meanwhile, some behaviors and lifestyles, including physical activity, cigarette smoking, and alcohol consumption, are considered to be associated with MetS in some studies, but the conclusions are inconsistent in other studies [9-14]. However, there have been few studies focusing on associated risk factors of MetS in Beijing. Therefore, it is important to know the latest prevalence and associated risk factors of MetS in order to formulate targeted policies to reduce the harmful consequences of MetS.

In 2017, we launched the Beijing Chronic Disease and Risk Factors Surveillance (BCDRFS), a population-based cross-sectional study of 13,259 participants aged from 18 to 79 years in 16 districts in Beijing. The BCDRFS focuses on the prevalence of MetS and its determinants in Beijing. In this report we estimated the weighted prevalence and main components of MetS and evaluated the associations of demographic factors and possible risk factors with the prevalence of MetS.

\section{Subjects and Methods}

\section{Study Design and Sample Population}

A multistage stratified cluster sampling method was used to obtain a representative sample of the Beijing residents aged 18-79 years. Only persons who had lived in their current residence for $\geq 6$ months in the past 12 months were eligible to participate. All 16 districts of Beijing city were covered; among them six are urban areas and the other are rural areas. The first stage of sampling was to select 1-10 townships from each district using the method of probability proportional to size. The second stage was to select three communities/villages from each chosen township by using the method of probability proportional to size. The third stage was to divide each community/village into several residential groups (at least 80 families), and one was selected from each chosen community/village by simple random sampling. Finally, one individual aged 18-79 years old was selected in each family by means of a Kish grid. The BCDRFS was conducted by the Beijing Center for Disease Prevention and Control (BJCDC) between August and November in 2017. The target sample size was 13,200,

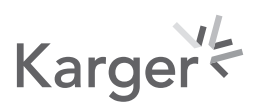


Ma et al.: Metabolic Syndrome in Beijing

and a total of 13,259 participants were recruited from 165 communities/villages. Finally, 13,240 people finished the entire survey, with a response rate of $99.9 \%$. After exclusion $(n=$ 283) due to missing information on major risk factors, 12,957 subjects were analyzed in this study.

\section{Training and Data Collection}

All study investigators were trained to be familiar with both the aims of the study and the specific tools and methods used. A standardized questionnaire was administered by trained investigators to obtain face-to-face information on demographic characteristics (age group and sex), socioeconomic factors (education level), place of residence (rural/urban area), and living habits such as physical activity, alcohol consumption, and smoking status. Waist circumference (WC) was measured at the midpoint between the last rib and the iliac crest using a tape measure with a precision of $1 \mathrm{~cm}$. BP was measured with the OMRON HEM1300 (OMRON, Dalian, China) three times on the left arm after the participant had been sitting at rest for at least $5 \mathrm{~min}$ and with $60 \mathrm{~s}$ between measurements with an observer present. The average of the three readings was used for analysis.

\section{Laboratory Measurements}

Participants were asked to fast for at least $10 \mathrm{~h}$ before venipuncture. A 5-mL overnight fasting blood sample was drawn into an EDTA vacutainer tube to measure serum lipids and glucose. Serum samples were obtained by centrifugation. The hexokinase method was used to test fasting blood glucose. The phosphoglycerol oxidase method was used to detect triglyceride (TG). The catalase method was used to detect high-density lipoprotein cholesterol (HDL-C). The detection instrument was a Hitachi 7600 automatic biochemical instrument.

\section{Outcome Definitions}

Current smokers were defined as participants who had smoked at least 100 cigarettes in their lifetimes and were still smoking. Harmful drinkers were defined as male participants who drank $\geq 61 \mathrm{~g}$ of alcohol per day or female participants who drank $\geq 41 \mathrm{~g} /$ day. The status of physical activity was obtained via the long International Physical Activity Questionnaire [15]. Insufficient physical activity was defined as $<150 \mathrm{~min}$ of moderate-intensity activity per week or equivalent [16]. The activity time of high-intensity physical activity times two was a fair amount of moderate-intensity activity time [17]. Seven to eight hours of sleep a night was regarded as appropriate sleep time, $<7 \mathrm{~h}$ or $>8 \mathrm{~h}$ were both inappropriate sleep time [18]. In addition, the waist-to-height ratio (WHtR) was calculated, and the number of 0.50 was used as cutoff based on the study of Ramírez-Vélez et al. [19]. Insufficient intake of vegetables and fruits was defined as $<400 \mathrm{~g}$ of vegetables and fruits per day [16].

The definition of MetS was based on the criteria of the International Diabetes Federation [20]. Central obesity was required as a mandatory component; it is defined at a WC $\geq 90 \mathrm{~cm}$ for men and $\geq 80 \mathrm{~cm}$ for women for the Chinese population. In addition, any two of the following four criteria needed to be met: (1) TG $\geq 1.7 \mathrm{mmol} / \mathrm{L}$; (2) HDL-C $<1.03 \mathrm{mmol} / \mathrm{L}$ in men and $<1.29 \mathrm{mmol} / \mathrm{L}$ in women; (3) systolic and/or diastolic BP $\geq 130 / 85 \mathrm{~mm} \mathrm{Hg}$, or treatment of previously diagnosed hypertension; (4) fasting plasma glucose (FPG) $\geq 5.6$ $\mathrm{mmol} / \mathrm{L}$ or previously diagnosed type 2 diabetes.

\section{Statistical Analysis}

Descriptive analysis was used to summarize the basic characteristics of the subjects. The weight was the product of the sampling weights and the post-stratification weights [21]. The data of the 6th national census in 2010 for Beijing residents were used to calculate the poststratification weights. The weighted prevalence and 95\% CI was calculated. The Rao-Scott

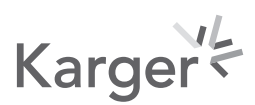


adjusted $\chi^{2}$ test was used to test differences in MetS and component rates, and complex sampling unconditional logistic regression was used to investigate associations between MetS (dependent variable) and demographic factors (including age, sex, and living area), behaviors (including current smoking, harmful use of alcohol, physical inactivity, inappropriate sleep time, and insufficient intake of vegetables and fruits), as well as measurement index (including WHtR). ORs with 95\% CIs were reported. Data analyses were carried out using IBM SPSS version 20.0.

\section{Results}

A total of 12,957 adults aged 18-79 years participated in the study (6,022 males, $46.48 \%$; $6,935$ females, $53.52 \%)$. The mean age of the participants was $44.78 \pm 15.47$ years (43.18 \pm 15.48 years for males and $46.17 \pm 15.32$ years for females). The distribution of participants according to age groups was as follows: $50.42 \%(n=6,533) 18-44$ years, $30.28 \%(n=3,924)$ $45-59$ years, and $19.30 \%(n=2,500) 60-79$ years. Among them, $65.10 \%(n=8,435)$ lived in urban areas.

The prevalence of MetS was $25.59 \%$ in total (Table 1) and was shown to be higher in men than in women (30.53 vs. $20.44 \%, p<0.01$ ). The highest MetS prevalence occurred in residents in the age group of $60-79$ years $(42.38 \% ; p<0.01)$ with an education level of primary school or below $(39.50 \% ; p<0.01)$. MetS showed higher prevalence in current smokers (31.43\%), harmful alcohol drinkers (44.03\%), people with inappropriate sleep time $(28.33 \%)$, or people with an unhealthy WHtR $(41.76 \% ; p<0.01)$. There was no statistical difference either between urban and rural area $(p>0.05)$, between people having enough vegetables and fruits or not $(p>0.05)$, or between subjects being physically inactivity or not $(p>0.05)$.

The proportion of central obesity, high FPG, high TG, low HDL-C, and high BP was 42.02, $27.96,32.87,27.25$, and $43.06 \%$, respectively. The top three components in men were high $\mathrm{BP}$, central obesity, and high TG, while the top three components in women were low HDL-C, central obesity, and high BP. The proportion of low HDL-C was higher in women than in men $(p<0.01)$, and the proportion of central obesity, high FPG, high TG, and high BP was higher in men than in women $(p<0.01)$.

The results from the complex sampling unconditional logistic regression analysis showed that the risk factors for having MetS included being 45-59 years old, being 60-79 years old, being male, living in a rural area, being educated with junior middle school level, being educated with primary school or below level, harmful use of alcohol, inappropriate sleep time, and having an unhealthy WHtR; the OR values were 1.55 (95\% CI 1.32-1.81), 1.94 (95\% CI 1.62-2.31), 1.51 (95\% CI 1.34-1.70), 1.27 (95\% CI 1.06-1.52), 1.38 (95\% CI 1.13-1.68), 1.44 (95\% CI 1.13-1.84), 1.50 (95\% CI 1.14-1.99), 1.23 (95\% CI 1.10-1.37), and 238.20 (95\% CI 92.54-613.12), respectively (Table 1).

About $21.20 \%$ (95\% CI 19.47-23.03) of the participants had no component of MetS, which was higher in women $(25.31 \%, 95 \%$ CI $23.29-27.45)$ than in men $(17.26 \%, 95 \%$ CI $15.26-19.47 ; p<0.01$ ). The proportion of those with three or more components was $29.60 \%$ (95\% CI 27.55-31.74) and higher in men (34.33\%, 95\% CI 31.70-37.07) than in women $(24.67 \%, 95 \%$ CI $22.43-27.04 ; p<0.001)$. The rest of the subjects $(49.20 \%$, 95\% CI $47.74-$ 50.65 ) had one or two components of MetS, with $48.41 \%$ (95\% CI 46.61-50.20) for males and $50.02 \%$ (95\% CI 48.22-51.82) for females ( $p>0.05)$. 


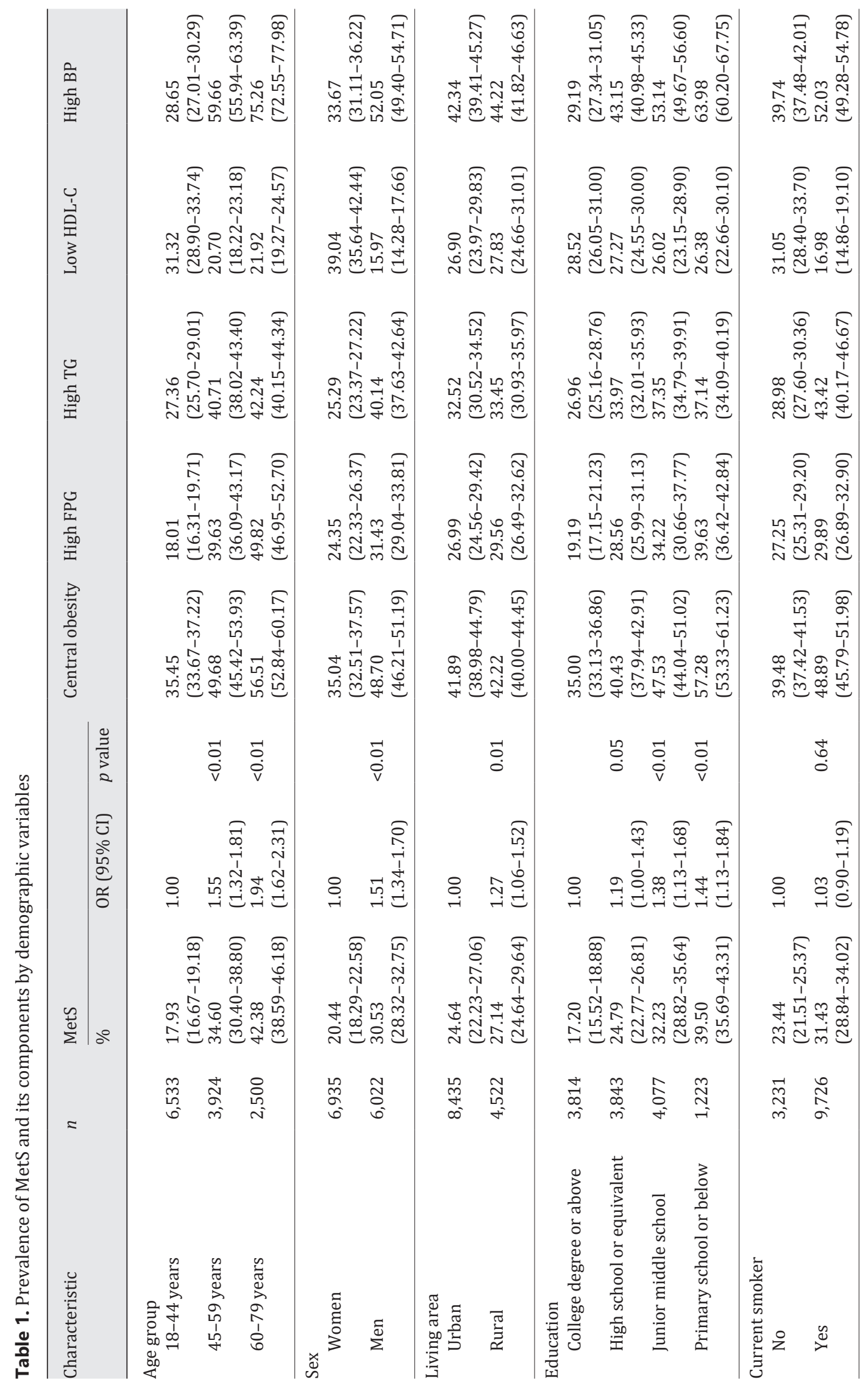




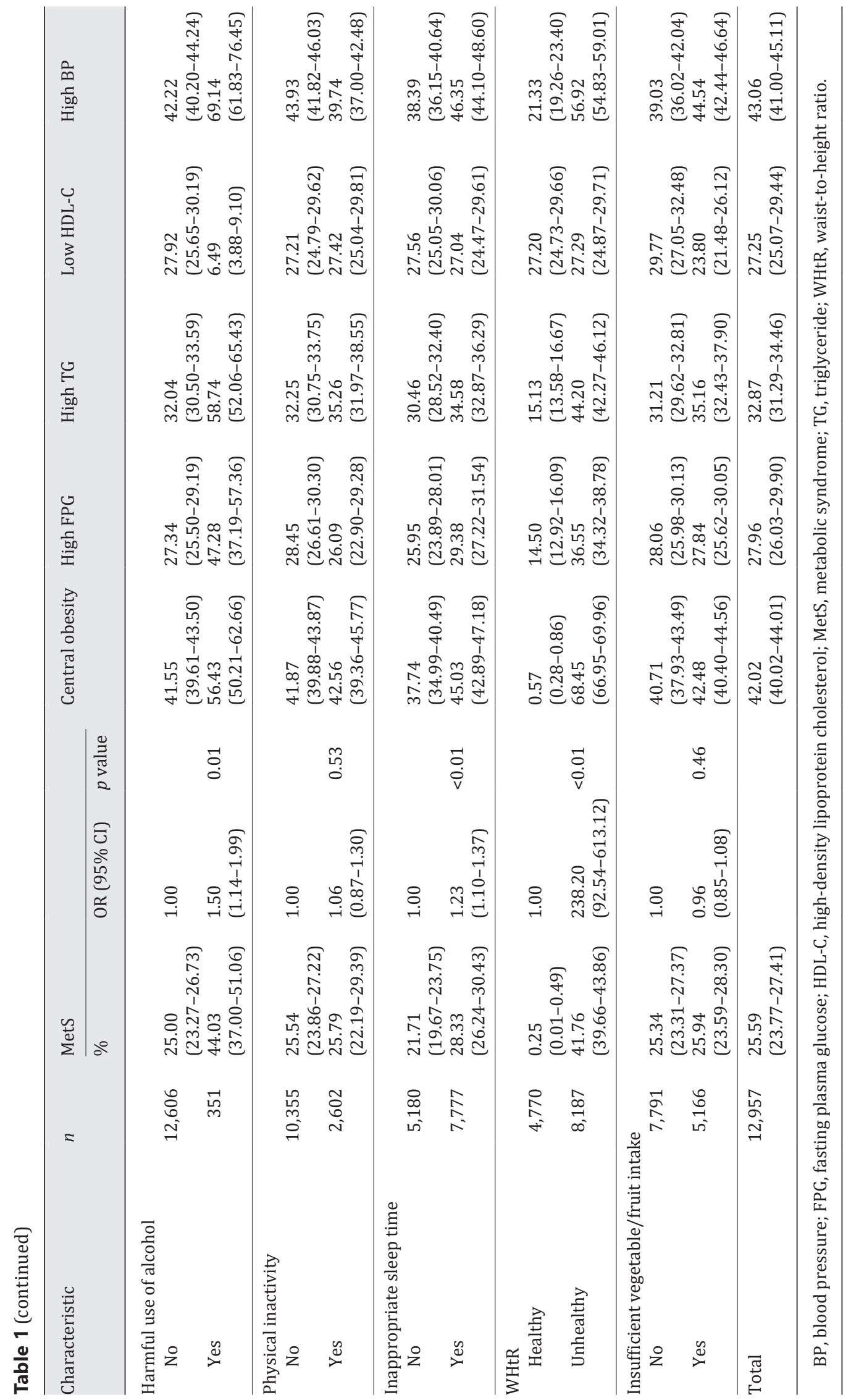


Ma et al.: Metabolic Syndrome in Beijing

\section{Discussion}

The prevalence of MetS in Beijing was 25.59\% in 2017 and lower than in Korea (31.3\%) [22] and Turkey (44.0\%) [23]. However, it had increased by $10.30 \%$ compared to that in 2005 (23.2\%) [7] and is still on the rise. Meanwhile, MetS components were predominantly prevalent. Only $17.26 \%$ of men and $25.31 \%$ of women in Beijing did not have any component of MetS, while $29.60 \%$ of subjects had three or more components. High BP was the first prevalent component in Beijing. However, the awareness, treatment, and control rate of hypertension in 2014 in Beijing were only 39.6, 33.6, and 12.8\%, respectively [24], which was significantly lower than that in the United States $(82.7,76.5$, and $54.1 \%$, respectively) [25]. Therefore, interventions for MetS and its components, especially BP, should be strengthened. Central obesity was the primary component for MetS and closely associated with diabetes mellitus. The abnormality rate was very high as $42.02 \%$. Thus, healthy WC should be maintained. Several studies have shown that WHtR was more closely related to some chronic diseases [26-28], and this ratio can reflect the accumulation of visceral fat as well as correct the effects of thoracic disease, scoliosis, and osteoporosis [29]. Complex sampling unconditional logistic regression results of this study showed that WHtR had a stronger relationship with MetS. Therefore, WHtR is more important to evaluate MetS. Beijing has launched a special action aiming to reduce salt, oil, and sugar intake and to keep healthy weight, mouth, and bones since 2017 [30]. This action should be further strengthened to obtain more effects on keeping healthy BP and blood glucose and maintaining healthy WHtR and WC.

Complex sampling unconditional logistic regression results showed that sex, age, living area, education level, inappropriate sleep time, and harmful use of alcohol were all associated with MetS. Personalized intervention should be promoted according to different population characteristics. MetS showed a higher prevalence in men and rural areas, which may be related to unhealthy lifestyle [8]. Studies showed that $<6 \mathrm{~h}$ of sleep was associated with elevated WC, and $>10 \mathrm{~h}$ of sleep was associated with MetS and TGs. Older adults with moderate sleep complaints can improve self-rated sleep quality by initiating a regular moderateintensity exercise program [31]. Therefore, good sleep should be kept by different ways such as enough moderate exercise. Harmful drinking of alcohol can increase the risk of hypertension and stroke. Dietary guidelines for Chinese residents suggest that the maximum recommended daily alcohol consumption for men and women is no more than 25 and $15 \mathrm{~g}$. In 2014, harmful use of alcohol for Beijing residents was 2.1\% [8]. To stay healthy, harmful alcohol drinkers should limit or abstain from alcohol. In 2017, the elderly aged $\geq 60$ years accounted for $24.7 \%$ of the registered population in Beijing, and the elderly aged $\geq 65$ years accounted for $16.4 \%$ [32]. With the aging of the population, the situation of MetS will become more serious. Our study showed that people with a high education level showed lower prevalence of MetS. Therefore, health education and promotion should be strengthened from childhood to form good lifestyle in order to prevent the occurrence of MetS and its components in adulthood.

This is a relatively large-scaled study in a Chinese population in Beijing. The standard questionnaire, physical examination, and laboratory test was implemented for each participant. However, there are still a few limitations to this study. First, its cross-sectional nature precluded causal assertions, so many findings from this study need further confirmation. Second, recall bias may have influenced the results, since the lifestyle and behaviors were all self-reported. To overcome this problem, all investigators were trained to get enough skills to support participant recall. Third, we did not find that current smoking, insufficient intake of vegetables and fruits, or physical inactivity were associated with MetS, which may need further research. At last, adults aged $\geq 80$ years were not included in this study, so the MetS risk factors of that population are unknown.

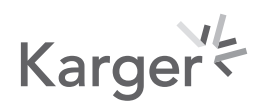




\section{Obesity Facts}

\section{Conclusions}

In summary, our findings indicate that the prevalence of MetS is still showing a rising trend in Beijing. Strategies aimed at the prevention and control of high BP should be prioritized to reduce the occurrence of MetS. WHtR is more important to evaluate MetS. Health education and personalized lifestyle intervention should be promoted to keep healthy WHtR and WC. An appropriate sleep time should be kept. Harmful alcohol drinkers should limit or abstain from alcohol.

\section{Acknowledgments}

The authors would like to thank all the participants in this study. Many thanks to the leadership and coordination work from the former Beijing Municipal Health Bureau and the contribution of staff in the centers for disease prevention and control and community health service from 16 districts in recruiting participants and investigation.

\section{Statement of Ethics}

This study was approved by the Ethics Review Board of the BJCDC (No. 5 of 2017). Written informed consent was obtained from each subject prior to data collection. All participants were aware that they could stop the interview at any time and refuse to answer questions without having to provide a reason.

\section{Conflict of Interest Statement}

The authors declare no conflict of interest.

\section{Funding Sources}

The BCDRFS was supported by National Key Research and Development Program of China (2019YFC1605100) and Beijing municipal financial funds.

\section{Author Contributions}

Z. Dong and A. Ma conceived and designed the study and revised the manuscript. A. Ma analyzed the data and drafted the manuscript. A. Ma, Z. Dong, K. Fang, and J. Dong performed the literature search and collected and cleaned the data. All authors read and approved the final manuscript.

\section{Karger'k}




\begin{tabular}{|c|c|}
\hline Obes Facts 2020;13:538-547 & \\
\hline DOI: 10.1159/000508842 & $\begin{array}{l}\text { (c) } 2020 \text { The Author(s). Published by S. Karger AG, Basel } \\
\text { www.karger.com/ofa }\end{array}$ \\
\hline
\end{tabular}

Ma et al.: Metabolic Syndrome in Beijing

\section{References}

1 Alberti KG, Zimmet PZ. Definition, diagnosis and classification of diabetes mellitus and its complications. Part 1: diagnosis and classification of diabetes mellitus provisional report of a WHO consultation. Diabet Med. 1998 Jul;15(7):539-53.

2 Zimmet P, Magliano D, Matsuzawa Y, Alberti G, Shaw J. The metabolic syndrome: a global public health problem and a new definition. J Atheroscler Thromb. 2005;12(6):295-300.

3 Lorenzo C, Okoloise M, Williams K, Stern MP, Haffner SM; San Antonio Heart Study. The metabolic syndrome as predictor of type 2 diabetes: the San Antonio heart study. Diabetes Care. 2003 Nov;26(11):3153-9.

4 Lakka HM, Laaksonen DE, Lakka TA, Niskanen LK, Kumpusalo E, Tuomilehto J, et al. The metabolic syndrome and total and cardiovascular disease mortality in middle-aged men. JAMA. 2002 Dec;288(21):2709-16.

5 McNeill AM, Rosamond WD, Girman CJ, Golden SH, Schmidt MI, East HE, et al. The metabolic syndrome and 11-year risk of incident cardiovascular disease in the atherosclerosis risk in communities study. Diabetes Care. 2005 Feb;28(2):385-90.

6 Expert Consultation WH; WHO Expert Consultation. Appropriate body-mass index for Asian populations and its implications for policy and intervention strategies. Lancet. 2004 Jan;363(9403):157-63.

7 Li G, de Courten M, Jiao S, Wang Y. Prevalence and characteristics of the metabolic syndrome among adults in Beijing, China. Asia Pac J Clin Nutr. 2010;19(1):98-102.

8 The People's Government of Beijing. The health and population health report in Beijing, 2014. Beijing: People's Medical Publishing House; 2015.

9 Wilsgaard T, Jacobsen BK. Lifestyle factors and incident metabolic syndrome. The Tromsø Study 1979-2001. Diabetes Res Clin Pract. 2007 Nov; 78(2):217-24.

10 Santos AC, Ebrahim S, Barros H. Alcohol intake, smoking, sleeping hours, physical activity and the metabolic syndrome. Prev Med. 2007 Apr;44(4):328-34.

11 Park YW, Zhu S, Palaniappan L, Heshka S, Carnethon MR, Heymsfield SB. The metabolic syndrome: prevalence and associated risk factor findings in the US population from the Third National Health and Nutrition Examination Survey, 1988-1994. Arch Intern Med. 2003 Feb;163(4):427-36.

12 Lakka TA, Laaksonen DE. Physical activity in prevention and treatment of the metabolic syndrome. Appl Physiol Nutr Metab. 2007 Feb;32(1):76-88.

13 Wada T, Urashima M, Fukumoto T. Risk of metabolic syndrome persists twenty years after the cessation of smoking. Intern Med. 2007;46(14):1079-82.

14 Wu S, Fisher-Hoch SP, Reininger B, McCormick JB. Recommended Levels of Physical Activity Are Associated with Reduced Risk of the Metabolic Syndrome in Mexican-Americans. PLoS One. 2016 Apr;11(4):e0152896.

15 Craig CL, Marshall AL, Sjöström M, Bauman AE, Booth ML, Ainsworth BE, et al. International Physical Activity Questionnaire: 12-country reliability and validity. Med Sci Sports Exerc. 2003 Aug;35(8):1381-95.

16 World Health Organization. NCD global monitoring framework. Geneva: World Health Organization; 2013. Available from: www.who.int/nmh/global_monitoring_framework/en/.

17 Ma AJ, Dong Z, Li H, Dong J, Fang K, Xie J, et al. Physical activity status among adults in Beijing, 2014. Cap J Public Health. 2017 Feb;11(1):26-8.

18 Chaput JP, McNeil J, Després JP, Bouchard C, Tremblay A. Seven to eight hours of sleep a night is associated with a lower prevalence of the metabolic syndrome and reduced overall cardiometabolic risk in adults. PLoS One. 2013 Sep;8(9):e72832.

19 Ramírez-Vélez R, Correa-Bautista JE, Martínez-Torres J, Méneses-Echavez JF, González-Ruiz K, GonzálezJiménez E, et al. LMS tables for waist circumference and waist-height ratio in Colombian adults: analysis of nationwide data 2010. Eur J Clin Nutr. 2016 Oct;70(10):1189-96.

20 Chinese Diabetes Society. China guideline for type 2 diabetes (2013). Beijing: Peking University Medical Press; 2014.

21 Ma AJ, Dong Z. Investigation of serum homocysteine levels and related risk factors of the elderly in Beijing. Chin J Prev Contr Dis. 2019 Jan;27(1):11-5.

22 Lim S, Shin H, Song JH, Kwak SH, Kang SM, Won Yoon J, et al. Increasing prevalence of metabolic syndrome in Korea: the Korean National Health and Nutrition Examination Survey for 1998-2007. Diabetes Care. 2011 Jun; 34(6):1323-8.

23 Gundogan K, Bayram F, Gedik V, Kaya A, Karaman A, Demir O, et al. Metabolic syndrome prevalence according to ATP III and IDF criteria and related factors in Turkish adults. Arch Med Sci. 2013 Apr;9(2):243-53.

24 The People's Government of Beijing. The health and population health report in Beijing, 2015. Beijing: People's Medical Publishing House; 2016.

25 Mozaffarian D, Benjamin EJ, Go AS, Arnett DK, Blaha MJ, Cushman M, et al.; Writing Group Members; American Heart Association Statistics Committee; Stroke Statistics Subcommittee. Heart Disease and Stroke Statistics - 2016 Update: A Report From the American Heart Association. Circulation. 2016 Jan;133(4):e38-360.

26 Ashwell M, Mayhew L, Richardson J, Rickayzen B. Waist-to-height ratio is more predictive of years of life lost than body mass index. PLoS One. 2014 Sep;9(9):e103483.

27 Silva MI, Lemos CC, Torres MR, Bregman R. Waist-to-height ratio: an accurate anthropometric index of abdominal adiposity and a predictor of high HOMA-IR values in nondialyzed chronic kidney disease patients. Nutrition. 2014 Mar;30(3):279-85. 
28 Xu Z, Qi X, Dahl AK, Xu W. Waist-to-height ratio is the best indicator for undiagnosed type 2 diabetes. Diabet Med. 2013 Jun;30(6):e201-7.

29 Chumlea WC, Baumgartner RN, Vellas BP. Anthropometry and body composition in the perspective of nutritional status in the elderly. Nutrition. 1991 Jan-Feb;7(1):57-60.

30 Zhao WH, Shi XM, Zhang J, Li KJ, Li Y, Tao MX, et al. Implementation strategies and scientific evidence for China Healthy Lifestyle for ALL. Chin J Prev Chron Dis. 2013 Jun;21(3):257-9.

31 King AC, Oman RF, Brassington GS, Bliwise DL, Haskell WL. Moderate-intensity exercise and self-rated quality of sleep in older adults. A randomized controlled trial. JAMA. 1997 Jan;277(1):32-7.

32 The People's Government of Beijing. The health and population health report in Beijing, 2017. Beijing: People's Medical Publishing House; 2018. 